\title{
Measuring structural parameters of crosslinked and entangled semiflexible polymer networks with single-filament tracing
}

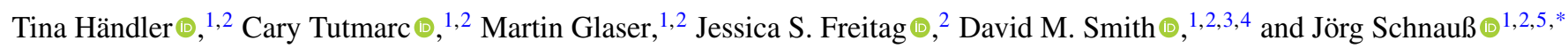 \\ ${ }^{1}$ Peter Debye Institute for Soft Matter Physics, University of Leipzig, Linnéstraße 5, 04103 Leipzig, Germany \\ ${ }^{2}$ Fraunhofer Institute for Cell Therapy and Immunology, Perlickstraße 1, 04103 Leipzig, Germany \\ ${ }^{3}$ Institute of Clinical Immunology, University of Leipzig Medical Faculty, 04103 Leipzig, Germany \\ ${ }^{4}$ Dhirubhai Ambani Institute of Information and Communication Technology, Gandhinagar 382 007, India \\ ${ }^{5}$ Unconventional Computing Laboratory, Department of Computer Science, University of the West of England, Bristol, United Kingdom
}

(Received 10 December 2020; accepted 17 April 2021; published 16 June 2021)

\begin{abstract}
Single-filament tracing has been a valuable tool to directly determine geometrical and mechanical properties of entangled polymer networks. However, systematically verifying how the stiffness of the tracer filament or its molecular interactions with the surrounding network impacts the measurement of these parameters has not been possible with the established experimental systems. Here we use mechanically programmable DNA nanotubes embedded in crosslinked and entangled F-actin networks, as well as in synthetic DNA networks, in order to measure fundamental, structural network properties like tube width and mesh size with respect to the stiffness of the tracers. While we confirm some predictions derived from models based purely on steric interactions, our results indicate that these models should be expanded to account for additional interfilament interactions, thereby describing the behavior of real polymer networks.
\end{abstract}

DOI: 10.1103/PhysRevE.103.062501

\section{INTRODUCTION}

Experimental and theoretical polymer physics study how features of the constituting elements determine the properties of whole polymer networks. Previous studies have examined interactions of semiflexible filaments [1] or investigated how geometrical parameters $[2,3]$ and bulk mechanical properties [4] can be deduced from tracking single filaments embedded in entangled networks. However, complex semiflexible polymer networks such as the cellular cytoskeleton contain multiple types of filaments with varied mechanical properties as well as crosslinkers creating physical connections between filaments [5]. Systematic investigations of the impact from these additional elements upon the dynamics of a single filament moving within the background network have not been possible with the systems typically used for reptation measurements. In our study, we use semiflexible DNA nanotubes with tunable stiffness [6] as embedded tracers in crosslinked and entangled F-actin networks and DNA nanotube networks [2]. Since the DNA filaments do not specifically interact with the network constituents, we are able to observe singlefilament dynamics and directly measure the tube width of both entangled and crosslinked F-actin, as well as DNA nanotube networks, with respect to tracer stiffness. By applying principles of the tube model [7], we determined the mesh size of

\footnotetext{
*Corresponding author: joerg.schnauss@ uni-leipzig.de
}

Published by the American Physical Society under the terms of the Creative Commons Attribution 4.0 International license. Further distribution of this work must maintain attribution to the author(s) and the published article's title, journal citation, and DOI. these polymer networks and confirmed some basic theoretical predictions such as the scaling of mesh size with monomer concentration. Although the concept has been proven to be successful in deriving scaling laws that are experimentally accessible [8-10], we find a need for adjustment beyond solely accounting for steric interactions, since the predictions dependent on tracer stiffness are not supported by our data. Nonetheless, our approach allowed us to study the architecture of crosslinked networks, opening new venues to investigate a broad range of filamentous networks by applying tube model predictions.

In this framework, the difficulties in describing semiflexible polymer networks as a many-body problem are reduced by studying a single test filament in the background of all other filaments [see Fig. 1(a)]. These background filaments constrain the test filament so that its effective available space is realized by a curved cylindrical tube [11], wherein the filament can reptate, i.e., diffuse along its contour [12]. The mesh size $\xi$ of a semiflexible polymer network is defined as the average distance between two filaments and, thus, depends on the monomer concentration of the solution, $\xi \propto 1 / c^{2}[9,13]$. We studied networks usually termed as concentrated solutions or tightly entangled semiflexible polymer networks, where the diameter $a$ of the reptation tube is assumed to be much smaller than the persistence length $l_{\mathrm{p}}$ of the constituting filaments, $a \lesssim \xi \ll l_{\mathrm{p}} \approx L[7,9,14-17]$, with $L$ denoting the filaments' contour length [see Fig. 1(a)]. Considering the relationship of tube width, mesh size, and persistence length, the same scaling law has been obtained by different argumentation, $a \propto \frac{\xi^{6 / 5}}{l_{\mathrm{p}}^{1 / 5}}[7,15,16]$.

This relation alone is insufficient to measure a network's mesh size quantitatively by detecting the tube width of 
(a)
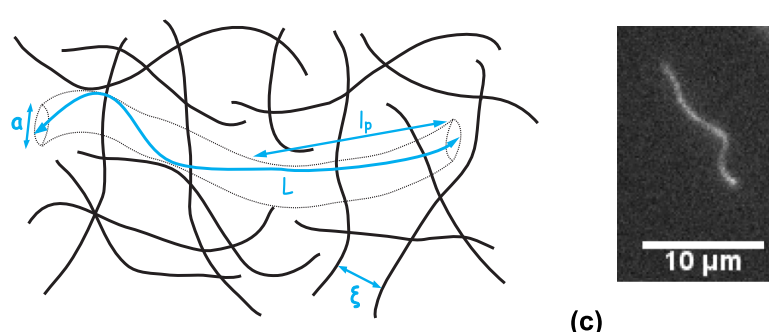

(c)

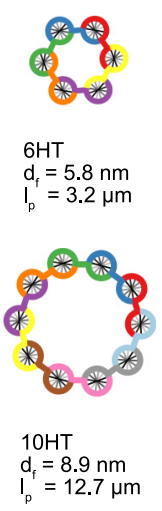

(b)

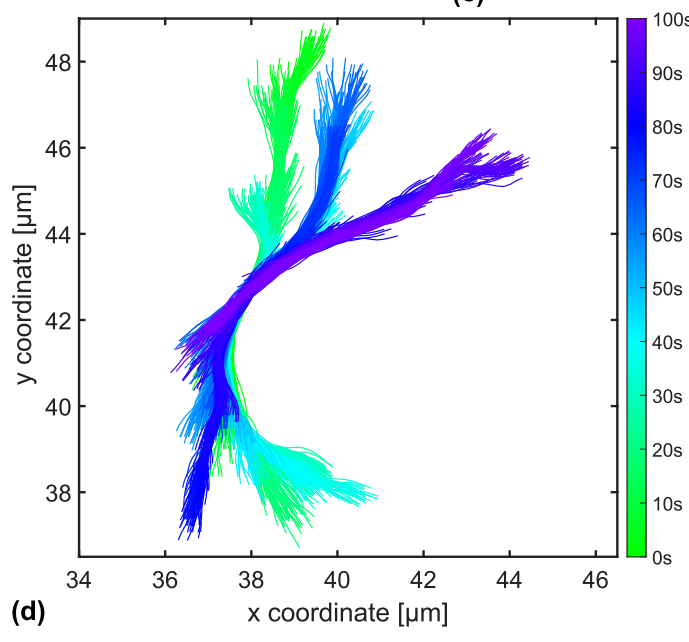

FIG. 1. (a) Scheme of a tracer filament of persistence length $l_{\mathrm{p}}$ and contour length $L$, confined to a reptation tube of width $a$ in a network of mesh size $\xi$. (b) Symbolic cross sections of the $n \mathrm{HT}$ tracer with the smallest (6HT) and largest (10HT) circumference used in this study, where each color corresponds to one of the 6 or 10 oligonucleotides (adapted from [2]). Filament diameters $d_{\mathrm{f}}$ and $l_{\mathrm{p}}$ of the other tracer filaments are in between the reported limits, see Appendix B. Since the typical contour lengths of $n$ HTs lie in the range of $2-15 \mu \mathrm{m}$ with averages around $6 \mu \mathrm{m}$ [6], they meet the definition $L \approx l_{\mathrm{p}}$ for semiflexibility. (c) Microscopy image of a fluorescently labeled 8HT filament. (d) Exemplary overlay of 1000 tracked frames of a reptating 8HT filament embedded in crosslinked F-actin. The color coding indicates the measurement time (see color map). The filament is neither crosslinked nor constrained to the original reptation tube, but able to explore the surrounding network during the observation time of $100 \mathrm{~s}$. Since the filament's fluctuations are rather strong at the ends, we selected only the middle part of each tube for determining the average tube width. Details are given in Appendix A.

embedded tracer filaments. Hinsch et al. [7] calculated a prefactor of 0.31 in a self-consistent treatment of the network by allowing fluctuations of the background filaments and decomposing the test filament into independent rods of appropriate length. They also found an additional term referring to boundary effects at the tube ends, using an expression for the free energy of a confined semiflexible polymer of finite length [18]. This second-order term accounts for the fact that short tracer filaments are more influenced by finite-length effects than long tracers and describes the effect on the measured tube width when finite filaments are considered, while the above mentioned scaling relation had only been derived for infinitely long tracer filaments. The complete expression reads [7]

$$
a \approx 0.31 \frac{\xi^{6 / 5}}{l_{\mathrm{p}}^{1 / 5}}+0.59 \frac{\xi^{2}}{L} .
$$

Equation (1) was originally derived for entangled polymer networks, but we were also able to employ it for determining the mesh size of crosslinked F-actin networks by using DNA nanotubes as embedded tracer filaments. These filaments have no binding sites for actin crosslinking complexes and are thus usable as decoupled, reptating tracers in crosslinked F-actin networks. DNA nanotubes have the additional benefit that their persistence length can be varied sufficiently to investigate theoretical predictions such as $a \propto l_{\mathrm{p}}^{-0.2}$, which were experimentally inaccessible before [2]. We measured the reptation of DNA tracer filaments $[6,19]$ with varying persistence length in different semiflexible polymer networks: entangled F-actin, crosslinked F-actin, and DNA nanotube networks. The results can be used to determine the tube width and mesh size, depending on the parameters tracer persistence length and contour length as well as polymer concentration.

\section{METHODS AND MATERIALS}

\section{A. DNA nanotubes as semiflexible tracer filaments}

DNA nanotubes were hybridized from $n$ partially complementary oligonucleotides as described in Ref. [20], forming $n$-helix tubes ( $n$ HTs) [see Fig. 1(b)]. Lyophilized oligonucleotides (biomers.net $\mathrm{GmbH}$, Germany) were resuspended in deionized water (sequences are given in $[2,6,20]$ ) according to the manufacturer. Oligonucleotide concentration was determined using a NanoDrop 1000 (Thermo Fisher Scientific Inc., USA). Final DNA $n$ HT samples consisted of the $n-1$ strands $\mathrm{U}_{1}-\mathrm{U}_{n-1}$ and one $\mathrm{T}_{n}$ strand, each at the same monomer concentration. For fluorescently labeled $n \mathrm{HTs}$ as tracer filaments, $\mathrm{U}_{1}$ was substituted by the modified $\mathrm{U}_{1}-\mathrm{Cy} 3$, with the dye $\mathrm{Cy} 3$ attached to the regular $\mathrm{U}_{1}$ strand [see Fig. 1(c)]. Buffer conditions for hybridization were $1 \times \mathrm{TE}(10 \mathrm{mM}$ Tris, $1 \mathrm{mM}$ EDTA, $p \mathrm{H} 8)$ and $12.5 \mathrm{mM}$ $\mathrm{MgCl}_{2}$. A thermocycler (TProfessional Standard PCR Thermocycler, Core Life Sciences Inc., USA) was used to hybridize the $n$ HTs. The hybridization protocol included randomization and dehybridization for $10 \mathrm{~min}$ at $90^{\circ} \mathrm{C}$ followed by complementary base pairing in 20 temperature steps of $-0.5^{\circ} \mathrm{C}$ for $60 \mathrm{~min}$ each, starting from $65^{\circ} \mathrm{C}$, and a quick drop to $20^{\circ} \mathrm{C}$. Depending on the chosen set of $n$ oligonucleotides, $n \mathrm{HTs}$ with different diameters and, accordingly, persistence lengths are formed [2,6,19,21], see Appendix B. Their contour length distribution is comparable to that of actin filaments, while the filament width stays below $10 \mathrm{~nm}$ [6]. After hybridization, $n$ HTs are stable for weeks and maintain their structure if they are kept below the structure's melting temperature of approximately $60^{\circ} \mathrm{C}$ [6]. Subsequently, we were able to polymerize F-actin background networks around the tracer filaments, ensuring them to be homogeneously distributed. In order to compare the reptation of $n \mathrm{HTs}$ in F-actin networks to the behavior of $n \mathrm{HTs}$ in nanotube networks, we chose $8 \mathrm{HT}$ as the constituting background filament since its persistence length of $8.9 \mu \mathrm{m}$ is comparable to that of actin filaments with $9 \mu \mathrm{m}$ [22]. Likewise, we used 8HT as tracers in F-actin background networks of similar persistence length.

\section{B. Actin}

G-actin was prepared from rabbit muscle as described previously [23]. Actin polymerization was initiated by the 
addition of 10 times concentrated F-Buffer $(1 \mathrm{M} \mathrm{KCl}$, $10 \mathrm{mM} \mathrm{MgCl} 2,2 \mathrm{mM}$ ATP, $10 \mathrm{mM}$ DTT, $20 \mathrm{mM}$ sodium phosphate, $p \mathrm{H}$ 7.5).

\section{DNA-based actin crosslinker wLX}

For the crosslinked F-actin background network, we selected the synthetic crosslinker wLX (weak LifeAct ${ }^{\circledR}$-based crosslinker) introduced by Lorenz et al. [24]. The crosslinker wLX was synthesized as described by Lorenz et al. [24]. In short, two actin-binding domains (LifeAct ${ }^{\circledR}$, Peptide Specialty Laboratories $\mathrm{GmbH}$, Germany) were covalently attached to the ends of double-stranded DNA (sequences given in [24]) using a copper-free click-chemistry approach. Since the two LifeAct ${ }^{\circledR}$ peptides were connected via double-stranded DNA, the composition transiently crosslinked actin filaments, forming networks that mechanically resemble networks of F-actin and the natural crosslinker $\alpha$-actinin [24]. We preferred using wLX over naturally occurring crosslinkers because of its reproducibility, i.e., defined length and binding strength, and applicability, as the fluorescent $n \mathrm{HT}$ tracers showed unspecific interactions with natural crosslinkers such as $\alpha$-actinin and heavy meromyosin (data not shown) that could be avoided by using wLX. The ratio of actin monomers to crosslinker molecules was chosen carefully to $150: 1$ so that the network was fully crosslinked, but not bundled [24].

\section{Sample preparation}

Prehybridized $n$ HTs labeled with the dye Cy3 were diluted gradually to $10 \mathrm{nM}$. For the F-actin network samples, Cy3labeled nanotubes were mixed with monomeric unlabeled actin (and additionally with the crosslinker wLX to a molar ratio of 150 actin monomers to 1 crosslinker molecule for the crosslinked actin networks) to an end ratio of nanotubes to actin filaments of at least 1:6000. Actin assembly was triggered by addition of $10 \times$ F-buffer and actin filaments were allowed to polymerize for $1-2 \mathrm{~h}$, ensuring that the F-actin network formed around the embedded tracer filaments. The samples were then placed between two glass slides previously passivated with $5 \%$ bovine serum albumin or Sigmacote (Sigma-Aldrich, USA), sealed with grease and left to settle for 30-60 min prior to measurement. Entangled actin networks were polymerized at $0.5 \mathrm{mgml}^{-1}$ and crosslinked networks at $0.3-0.9 \mathrm{mgml}^{-1}$.

For the 8HT network samples, a small fraction of diluted, labeled $n \mathrm{HT}$ was gently mixed with a prehybridized unlabeled 8HT network to a final ratio of 1:4000 of labeled to unlabeled filaments [20]. Subsequently, the sample solution was placed between two glass slides previously coated with Sigmacote (Sigma-Aldrich, USA) and sealed with grease and nail polish. 8HT network samples were equilibrated overnight at $4{ }^{\circ} \mathrm{C}$ and measured at $8 \mu \mathrm{M}$.

\section{E. Measurement and data analysis}

The reptation of fluorescently labeled $n \mathrm{HT}$ tracers in different background networks was recorded via an epifluorescence microscope (Leica DM-IRB, 100× objective, NA 1.35) with an attached CCD camera (Andor, iXon DV887) at a frame rate of $10 \mathrm{~Hz}$ and tracked with the ImageJ plugin

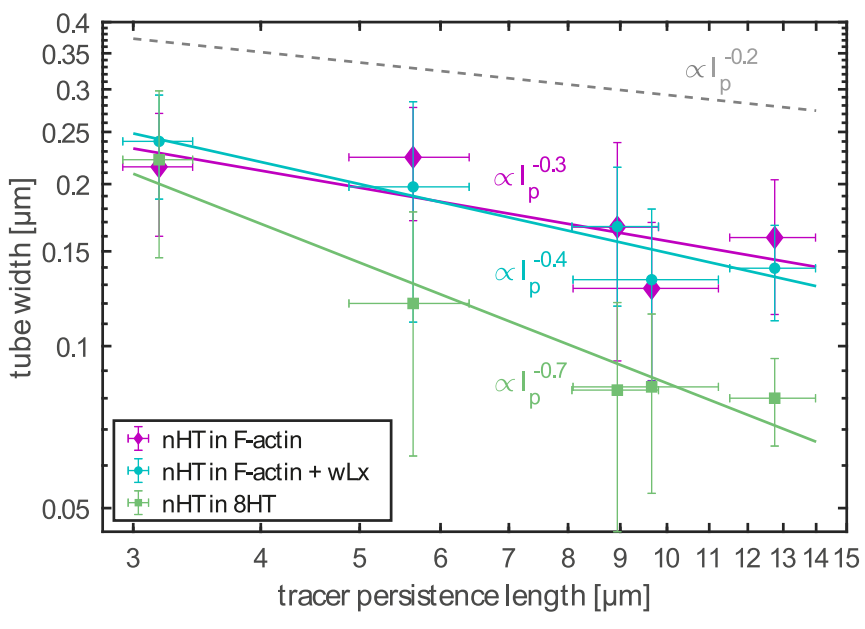

FIG. 2. Using DNA nanotubes as tracer filaments, we were able to examine the dependency of tube width on persistence length for three different background networks: entangled F-actin networks (purple diamonds), crosslinked F-actin networks (crosslinked at a ratio of actin monomers to wLX of 150:1 [24], blue circles), and $8 \mathrm{HT}$ networks (green squares). Tube width error bars are weighted standard errors obtained by calculating weighted averages of several individual tracer filaments. The tracers were $6 \mathrm{HT}, 7 \mathrm{HT}, 8 \mathrm{HT}, 9 \mathrm{HT}$, and $10 \mathrm{HT}$, with a persistence length range from approximately $3-$ $13 \mu \mathrm{m}$ [2], see Appendix B. The dashed line indicates the theoretical scaling derived from the tube model. Matching colored lines depict the power laws resulting from fitting. To access the scaling between tube width and persistence length, it is necessary to include data from tracer filaments of varying persistence length. Naturally, these tracers differ in contour length, therefore we only used the leading term of Eq. (1) to fit the data presented here and omitted the second term that involves the tracer's individual contour length.

JFilament [25], see Figs. 1(c) and 1(d). Subsequently, the tracking data were used to determine the tube width and mesh size data by analyzing multiple subsets of 100 frames for each individual tracer filament. Details on the data processing are given in Appendix A.

\section{RESULTS}

\section{A. Tube width scaling with persistence length}

After calculating a weighted mean for all tube widths measured with one type of DNA nanotube tracer, we determined the power law exponents of the tube width scaling with the tracers' persistence length. Figure 2 shows the measured tube width values for five types of $n \mathrm{HT}$ filaments in three distinct background networks.

One might expect to observe different tube widths for entangled and crosslinked networks of the same concentration since some fluctuation modes of the surrounding polymers are suppressed in crosslinked networks [26]. However, we observe nearly the same values for tube widths in entangled and crosslinked F-actin networks, indicating that the crosslinker wLX does not change the network geometry for the chosen ratio of actin monomers to crosslinker molecules [24]. In 8HT networks, $n$ HTs were less motile than in F-actin networks and appeared nearly stuck, resulting in lower tube widths. 


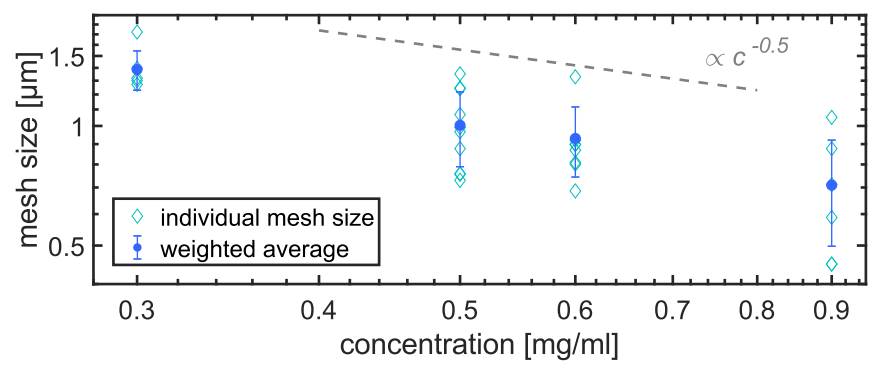

FIG. 3. Mesh size vs monomer concentration for F-actin networks crosslinked with wLX. Since each measured tube width that enters the calculation of the mesh size is only a measure of the local constraint of the individual tracer filament, we measured several tracers per actin monomer concentration and averaged the results. Error bars are calculated from relative error propagation, see Appendix A. The dashed line indicates the expected scaling $\xi \propto c^{-0.5}[9,13]$.

The predicted scaling $a \propto l_{\mathrm{p}}^{-0.2}$ (indicated by the dashed line in Fig. 2) could not be confirmed in all of the three background networks. It is only observed for $n$ HTs in entangled F-actin where the exponent is $-0.33 \pm 0.16$ so that an exponent of -0.2 is within the error limits. The power law exponent for crosslinked F-actin is $-0.42 \pm 0.08$. However, since the tube width values have relatively large errors, it cannot be concluded that the predicted scaling does not apply here. The scaling exponent for the 8HT network is $-0.74 \pm$ 0.12 which differs from the predicted exponent. Together with the observed lower motility of $n$ HTs in $8 \mathrm{HT}$ background, this hints at unspecific interactions between filaments that are not captured by the tube model.

By fitting the leading term of Eq. (1), we were also able to derive the mesh size of the background networks. The estimated mesh sizes are $1 \mu \mathrm{m}$ for entangled F-actin, $1.2 \mu \mathrm{m}$ for crosslinked F-actin, and $1.4 \mu \mathrm{m}$ for 8HT networks, corresponding well with previous publications [2,27].

\section{B. Mesh size of crosslinked F-actin networks}

As demonstrated in this study, the measured tube width is dependent on tracer properties such as the persistence and contour length, as well as, on the local constraint exerted by the surrounding polymers on the tracer. According to theory, an intrinsic property of the network is the mesh size, which, therefore, makes it a desirable quantity to measure. With our DNA nanotube tracers, we were able to measure the mesh size of crosslinked F-actin networks. This is not possible using actin-based tracers because they do not reptate when actin crosslinkers are present. 8HTs were chosen for their persistence length comparability to unstabilized actin filaments [22], avoiding effects stemming from different polymer stiffnesses. By measuring the tube width of embedded 8HTs and employing Eq. (1) with the adjusted scaling of $a \propto l_{\mathrm{p}}^{-0.42}$ obtained from the fit plotted in Fig. 2, we determined the mesh size for crosslinked F-actin networks of four different concentrations (see Fig. 3). The expected scaling $\xi \propto c^{-0.5}$ $[9,13]$ was confirmed as it had already been for entangled F-actin [2,28]. The resulting mesh size of $1 \pm 0.2 \mu \mathrm{m}$ for a concentration of $0.5 \mathrm{mgml}^{-1}$ is comparable to the fitted mesh size of crosslinked F-actin networks from Fig. 2 and to already
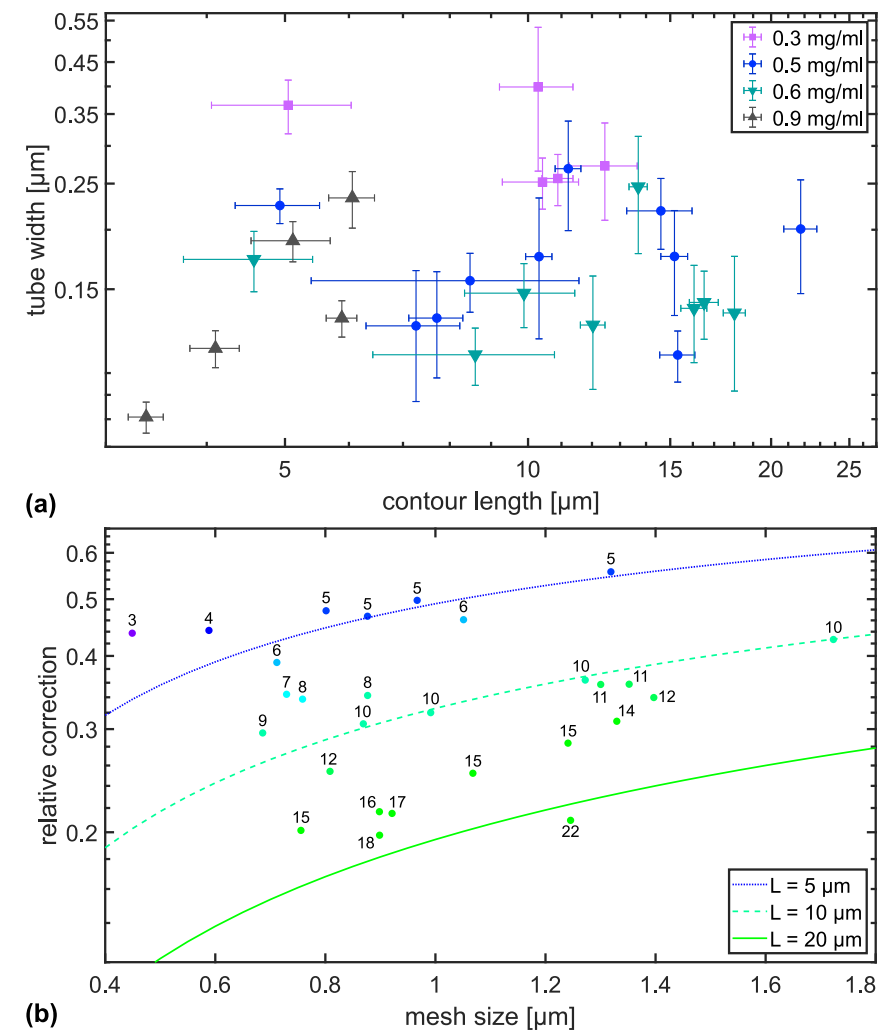

FIG. 4. (a) Individual tube widths for 8HTs in crosslinked F-actin background networks are plotted over the tracers' contour lengths. There is no evidence of a clear scaling relation between tube width and contour length, which is in direct contrast to theoretical predictions. The color coding refers to the networks' actin monomer concentration. Error bars are standard errors, see Appendix A. (b) This plot shows the relative correction $\left(0.59 \frac{\xi^{2}}{L}\right) \frac{1}{a}$ versus mesh size (circles, calculated by $a \propto 0.31 \frac{\xi^{6 / 5}}{l_{\mathrm{p}}^{0.42}}+0.59 \frac{\xi^{2}}{L}$ ) for the data of (a). The numbers accompanying each data point are the filaments' rounded contour lengths. Predictive curves of relative corrections are plotted for theoretical contour lengths of 5, 10, and $20 \mu \mathrm{m}$. The colors of data points and predictive curves are matched to illustrate the gradually changing contour length.

published values for entangled F-actin of the same concentration [2,27], implying that crosslinking does not change the geometry of the F-actin network.

\section{Effect of finite tracer length}

Since filaments with a shorter contour length are presumably more affected by boundary effects at the tube ends, we examined whether the scaling of the second-order term $a \propto L^{-1}$ of Eq. (1) applies to our data. We chose to study this by measuring tube widths and contour lengths of 8HTs embedded in crosslinked $\mathrm{F}$-actin background networks at various concentrations. In Fig. 4(a) all resulting tube widths for 8HTs are plotted over the tracers' contour lengths. The predicted scaling $a \propto L^{-1}$ is not fulfilled. However, this does not mean that the filaments are not affected by the predicted boundary effects. The leading term of Eq. (1), by itself, is valid for 
infinite tracer filaments; the second term corrects for polydisperse tracers [7].

To quantify the effect of such finite tracer lengths, we computed the relative correction of the tube width stemming from the second term of Eq. (1). The results plotted in Fig. 4(b) reveal that the relative correction is indeed higher for shorter filaments. Figure 4(b) includes calculated curves of relative corrections for distinct contour lengths analogous to predictions from Hinsch et al. [7]. The data points match these predictions well, illustrating that longer filaments are less affected by boundary affects at the end of the tube. However, the observed effect of these relative corrections of tube widths is not reflected in the variation of the absolute values of tube widths, probably because local inhomogeneities affecting each individual tracer's observed tube width have a higher influence than the finite-length effect.

The results presented in Fig. 4 are obtained for 8HT tracers in crosslinked F-actin, where the tracer persistence lengths equal that of the surrounding filaments. This can also be reproduced for all other datasets of embedded $n \mathrm{HT}$ tracers, see Appendix C.

\section{DISCUSSION}

Although a difference between the tube widths of entangled and crosslinked F-actin networks at the same concentration might be possible due to the suppression of fluctuation modes of crosslinked background filaments, the average values were comparable within the error bars, indicating that the fluctuations of surrounding filaments do not have a high impact on the constraining potential exerted on the tracer filament [3,29], at least not on the timescales used for determining the tube diameter. By varying the tracers' persistence length, we found that the expected scaling relation $a \propto l_{\mathrm{p}}^{-0.2}$ is not valid for all of the three examined background networks. 8HT background networks show a different relation between tube width and persistence length, presumably due to interactions between $n \mathrm{HT}$ tracers and the $8 \mathrm{HT}$ network that are not accounted for in the general reptation model, e.g., electrostatic interactions between the DNA molecules that lead to an effective stickiness [30]. Recent studies have shown that other scaling predictions made from the tube model are not applicable to networks of $n$ HTs [2] and that sticky interactions lead to altered bulk properties of semiflexible polymer networks [31,32]. The interactions between different components of the cytoskeleton have been revealed to affect the network architecture as well, which in turn influences mechanical properties $[32,33]$. We speculate that an experimental adjustment of the exponent in the relation $a \propto l_{\mathrm{p}}^{-0.2}$ is acceptable when the interactions not included in the tube model only mildly affect the tracers' reptation.

Previous publications examining reptation have made use of F-actin tracers in entangled F-actin [1,34]. Stiff filaments [35] and flexible tracers $[3,4]$ have also been measured. There is no experimental study that tests the predicted scaling $a \propto$ $l_{\mathrm{p}}^{-0.2}$ for semiflexible filaments over a broad range of $l_{\mathrm{p}}$. A recent attempt by Keshavarz et al. [3] utilized flexible filaments with different persistence lengths, but was limited to two data points, hindering a clear verification of a scaling law. However, the same study proved the prediction $a \propto \xi^{6 / 5}$ for flexible filaments where $\xi$ can be calculated independently of $a$ from the elastic plateau modulus obtained by rheological measurements [3]. Consequently, we were able to measure the mesh size of crosslinked F-actin networks by applying Eq. (1). Until now, this had only been done before for entangled F-actin by employing actin filaments as tracers $[2,27]$. The results for crosslinked F-actin networks reveal a good agreement with the established concentration scaling $\xi \propto c^{-0.5}[2,28]$ and the absolute values are well comparable to those published for entangled F-actin networks [2,27], confirming that the crosslinker wLX does not change the network architecture for the chosen crosslinker concentration [24] and indicating that suppressing background filament fluctuations does not have a high impact on the constraining potential exerted on the tracer filament $[3,29]$. Our findings might be compared to bulk measurements, e.g., by performing scattering experiments on crosslinked and entangled F-actin, since formulations for scattering functions for semiflexible polymers in different environments and under constraints already exist [36,37].

The correspondence of measured mesh size values between entangled and crosslinked F-actin networks justifies the use of Eq. (1) for the crosslinked networks, even if it was derived for an entangled network with fluctuating background filaments. This is comparable to the findings of Gardel et al. [38], where the elastic moduli of crosslinked F-actin networks followed the concentration scaling that had been deduced for entangled networks in the tube model framework previously [8]. We would like to point out that the use of other actin crosslinkers might change the network architecture or interactions between background network and tracer filaments in such a way that it is not possible to use Eq. (1) for data evaluation. The effect of suppressed fluctuations stemming from crosslinkers could be modeled by incorporating an additional potential into the tube theory, which vanishes in the limit of purely entangled solutions and becomes stronger when more degrees of freedom are reduced.

A recent simulation study investigated the difference between static and entangled background polymers and its effect on stress relaxation in the framework of the tube model [39]. Lang and Frey [39] showed that constraint release, the dissolution of the reptation tube upon correlated motion of the tracer and its surrounding filaments, leads to altered tracer dynamics and relaxation times $[4,9,39]$. This could explain why conventional tube model predictions do not fully account for the examined networks, which differ from a structure with fixed obstacles.

\section{CONCLUSION}

We have tested the persistence length dependency of tracer filaments reptating in a polymer network, revealing that the tube model needs to be extended to describe actual experimental data, where more than steric interactions are present. Additionally, we showed that networks with finite-length polymers differ from theoretical solutions with infinitely long filaments. Our results may guide theoreticians to develop a comprehensive framework for both entangled and crosslinked polymer networks. However, the tube model does indeed provide valid predictions for measuring parameters describing 
the geometrical structure. Utilizing mechanically tunable filaments as tracers, not only entangled, but also crosslinked networks can be architecturally characterized. This approach is particularly appealing because it avoids additional influences inherent to embedding fluorescent beads for these determinations $[28,40]$ or labeling entire networks with fluorophores [41]. Our method may also prove applicable to other semiflexible polymer networks, e.g., networks of other cytoskeletal filaments or collagen fibers that are physically crosslinked. The programmability of DNA nanotubes further allows one to choose a suitable tracer's persistence length to match that of the background network of interest, thus mitigating emergent effects due to different polymer stiffnesses. Furthermore, stable and biocompatible probe filaments like DNA nanotubes may be utilized to measure local properties of heterogeneous scaffolds such as the extracellular matrix or the cellular cytoskeleton.

\section{ACKNOWLEDGMENTS}

We thank Josef A. Käs for fruitful discussions. We acknowledge funding by the European Research Council (ERC-741350). C.T. acknowledges funding from the European Social Fund (ESF-100380880).

T.H. and C.T. contributed equally to this work.

\section{APPENDIX A: TUBE WIDTH ANALYSIS}

After preprocessing the image stacks with the ImageJ plugin Stack Contrast Adjustment [42], filament backbones were determined by the ImageJ plugin JFilament [25]. From the backbones, tube widths were derived using a self-written Matlab (The MathWorks, Inc.) script as depicted by Fig. 5. The process is as follows: Fig. 5(a) plots all 1000 tracked configurations of the filament during the observation time of $100 \mathrm{~s}$ (see color bar at side). From these 1000 frames, three sets of 100 consecutive frames are chosen so that the filament stays in one reptation tube for the selected $10 \mathrm{~s}$. As a criterion, the deviation of the filament's midpoint from the reference midpoint of the first frame is examined as displayed in Fig. 5(c). Figure 5(b) shows an example of one chosen set of 100 tracked configurations (blue). The gray dotted line depicts the mean of all 100 configurations and the red lines are perpendicular to this tube backbone. To determine the tube width, the intersection points of the 100 individual configurations with the orthogonal lines are detected. The resulting intersection positions (orange crosses) are processed for each tube backbone point as shown in Fig. 5(d): From the intersection positions, a kernel density distribution is calculated (red). A Gaussian distribution (blue) is fitted to this kernel density estimate. Since this process is repeated for every orthogonal line from Fig. 5(b), we can plot a tube width measure for each point of the tube backbone as in Fig. 5(e). The orange curve shows the distance of the two outermost individual configurations, whereas the blue line is the standard deviation of the Gaussian fit from Fig. 5(d). Due to a rather high fluctuation of the filament's ends, only the middle part of the curve in Fig. 5(e) is used (i.e., tube arc length from 1 to $5.6 \mu \mathrm{m}$ for this example) and the resulting tube width of the chosen set is the average of two times the standard deviation of the Gaussian fit.
TABLE I. Characteristic lengths of the tracer filaments $[2,6], l_{\mathrm{p}}$ is the persistence length and $d_{\mathrm{f}}$ is the tracer thickness.

\begin{tabular}{lcc}
\hline \hline Tracer & $l_{\mathrm{p}}(\mu \mathrm{m})$ & $d_{\mathrm{f}}(\mathrm{nm})$ \\
\hline $6 \mathrm{HT}$ & $3.18 \pm 0.25$ & 5.7 \\
7HT & $5.64 \pm 0.76$ & 6.7 \\
8HT & $8.94 \pm 0.87$ & 7.6 \\
9HT & $9.66 \pm 1.57$ & 8.6 \\
$10 \mathrm{HT}$ & $12.75 \pm 1.23$ & 9.5 \\
\hline \hline
\end{tabular}

The tube width of the whole filament is defined as the average of the tube width from the three subsets of configurations, the standard error of the tube width $u(a)$ is the standard error of this mean. To implicitly calculate the mesh size from

$$
a \approx 0.31 \frac{\xi^{6 / 5}}{l_{\mathrm{p}}^{1 / 5}}+0.59 \frac{\xi^{2}}{L},
$$

the contour length of the filament was deduced as the average contour length of the 300 frames used for tube width determination. Analogously to the tube width, the standard error $u(L)$ of the contour length is the standard error of this mean. For the mesh size we calculated the error $u(\xi)$ by relative error propagation using $u(\xi)=u\left(l_{\mathrm{p}}\right) \epsilon 5 / 6+u(a) 5 / 6+u(L) / 2$, with $u\left(l_{\mathrm{p}}\right)$ being the standard error of the persistence length according to Table I. The factor $\epsilon$ is the exponent extracted from the fit displayed in Fig. 2, i.e., $\epsilon=0.33$ for entangled F-actin, $\epsilon=0.42$ for crosslinked F-actin, and $\epsilon=0.74$ for the $8 \mathrm{HT}$ background network.

\section{APPENDIX B: CHARACTERISTIC LENGTHS OF $n$ HTs}

In Table I we give an overview of the $n \mathrm{HT}$ persistence lengths $l_{\mathrm{p}}$ and filament diameters $d_{\mathrm{f}}$ used throughout our calculations. Filament diameters were estimated from $n \mathrm{HT}$ circumference values given in Ref. [6]. Persistence length values were taken from Ref. [2], except for 7HT, where we measured the persistence length with the method described in [2]. Since the persistence length of actin filaments is about $9 \mu \mathrm{m}$ [22], approximate ratios between tracer $(6 \mathrm{HT}$, $7 \mathrm{HT}, 8 \mathrm{HT}, 9 \mathrm{HT}$, and 10HT) and background filament $(\mathrm{F}-$ actin or $8 \mathrm{HT})$ persistence lengths are $3 \times, 1.5 \times, 1 \times, 1 \times$, and $(1 / 1.5) \times$, respectively.

\section{APPENDIX C: CONTOUR LENGTH CORRECTIONS}

We used Eq. (A1) to calculate mesh sizes from measured tube widths. The second term accounts for boundary effects occurring at the ends of the reptation tube. In the main text, we showed that the scaling $a \propto L^{-1}$ is not observed for 8HT tracers embedded in F-actin networks crosslinked with wLX at different concentrations [see Fig. 4(a)]. In Fig. 6 we plot individual tube widths vs contour lengths of five $n \mathrm{HT}$ tracer types in three different background networks with constant concentrations. The results do not support the predicted scaling $a \propto L^{-1}$ as shown before, again confirming that the variation of the absolute values of tube width due to finite length effects is negligible. By calculating the relative correction of the tube width stemming from finite length effects, we are able to 

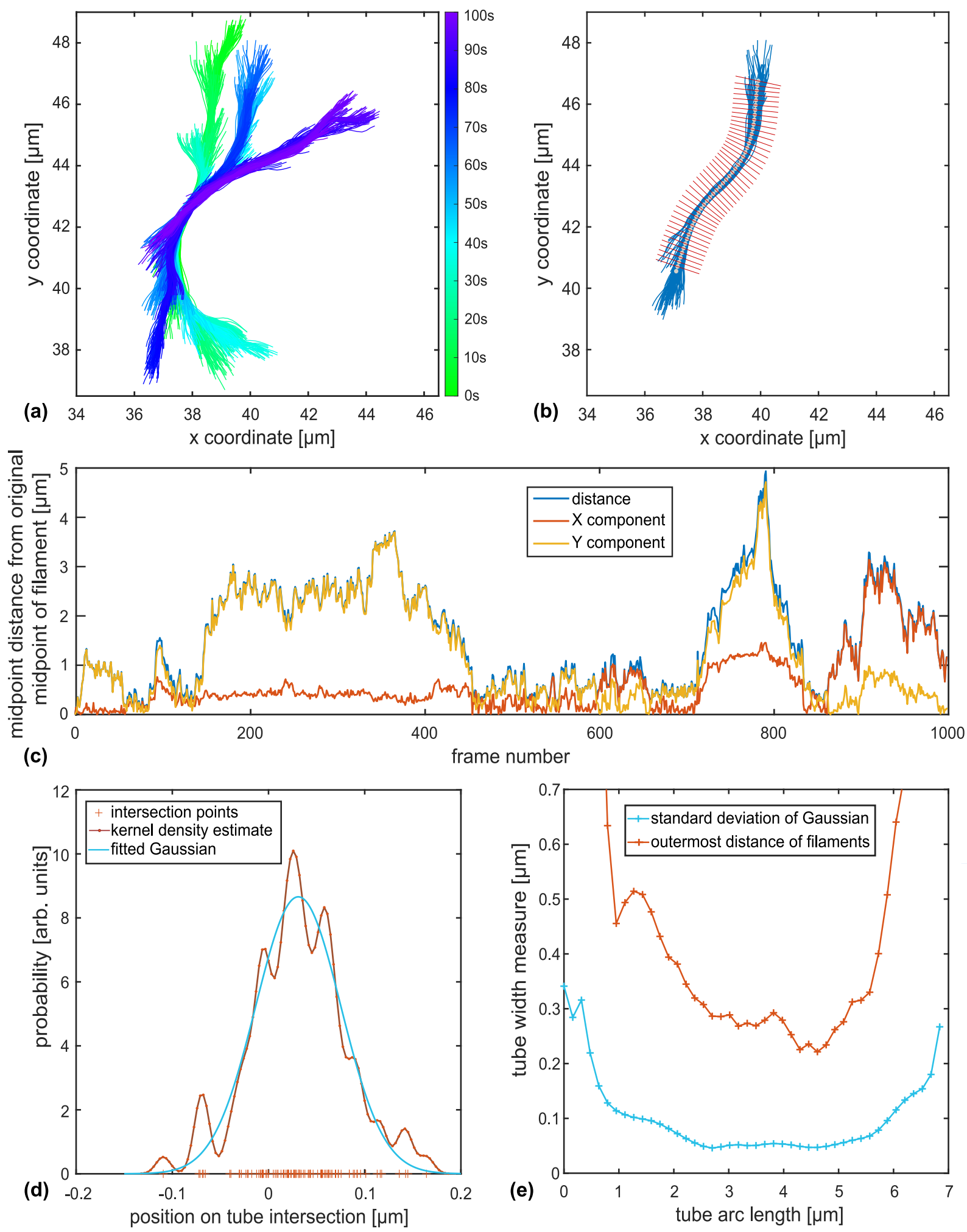

FIG. 5. Scheme of tube width determination, example filament is an $8 \mathrm{HT}$ in F-actin at $0.5 \mathrm{mgml}^{-1}$ crosslinked with wLX at a ratio of 150:1. The process is explained in detail in the text.

verify that these effects are small, but larger for shorter tracer filaments [see Fig. 4(b)]. Here we show that the same results are obtained for all measured combinations of tracer filaments and background networks. In Fig. 7 the relative correction $\left(0.59 \frac{\xi^{2}}{L}\right) \frac{1}{a}$ is plotted against the mesh size determined by $a=0.31 \frac{\xi^{6 / 5}}{l_{\mathfrak{p}}}+0.59 \frac{\xi^{2}}{L}$ with an exponent $\epsilon=0.33$ for entangled F-actin, $\epsilon=0.42$ for crosslinked F-actin, and $\epsilon=0.74$ for the $8 \mathrm{HT}$ network, as extracted from the fit displayed in Fig. 2. 

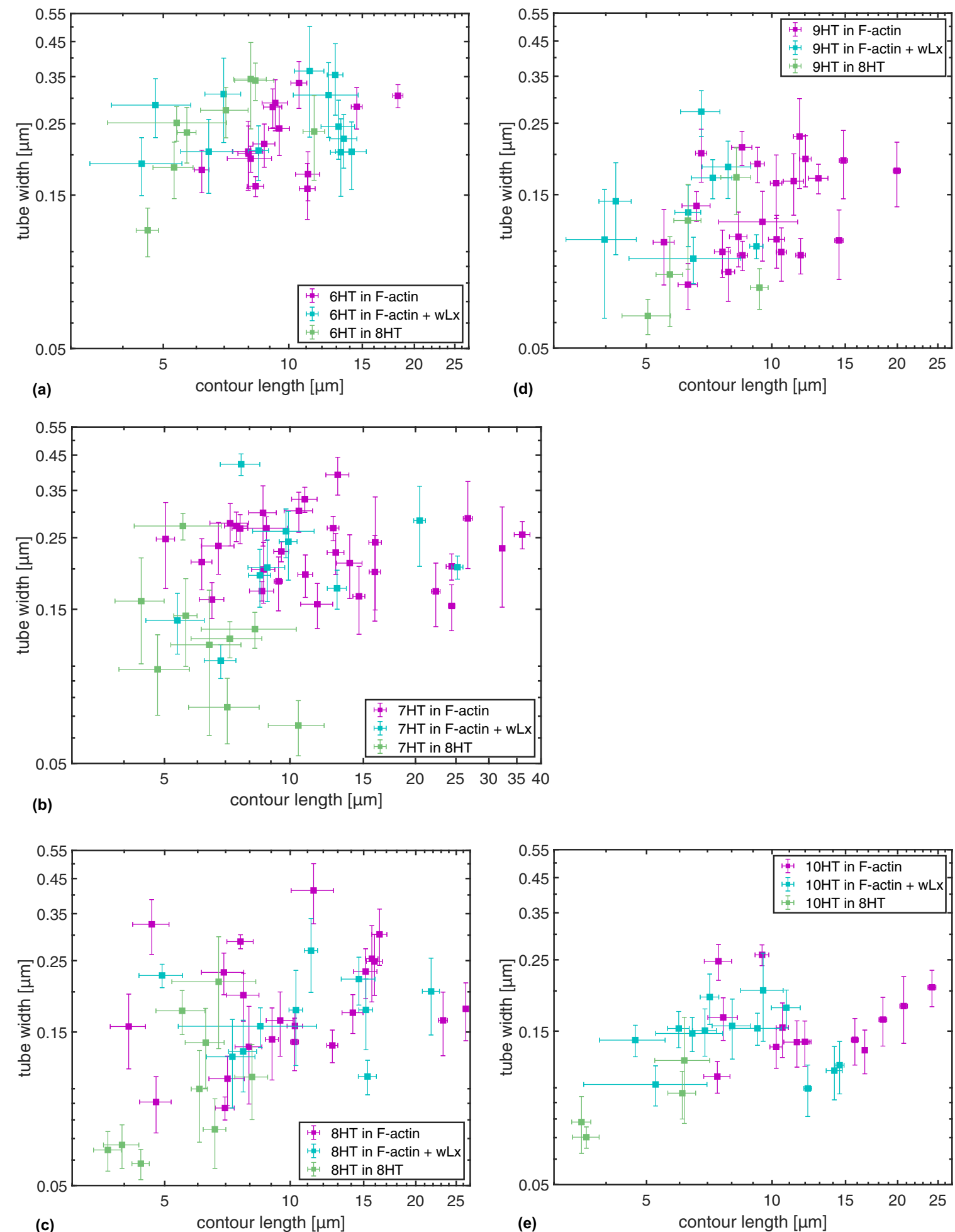

FIG. 6. Tube width vs contour length for all individual tracer filaments which are averaged in Fig. 1. The predicted scaling $a \propto L^{-1}$ is not observed for 6HT (a), 7HT (b), 8HT (c), 9HT (d), and 10HT (e) in three different background networks. 

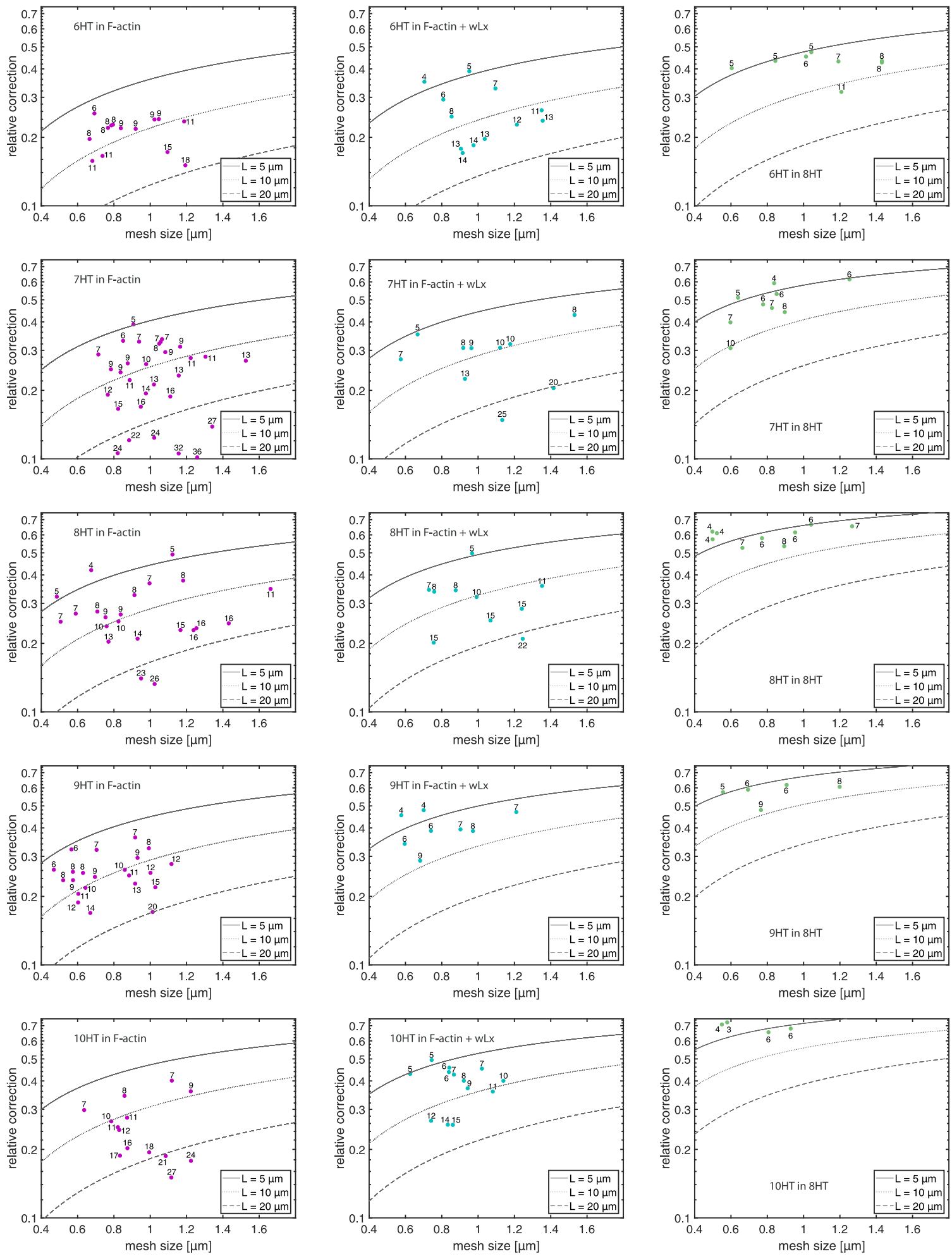

FIG. 7. Relative tube width correction vs calculated mesh size for all data sets of different $n$ HTs (varying from top to bottom) embedded in three background networks (varying from left to right). The curves for 5, 10, and $20 \mu \mathrm{m}$ contour length are predictions, while the colored dots are data points, each accompanied by the rounded contour length of the respective tracer filament. 
[1] J. Käs, H. Strey, and E. Sackmann, Direct imaging of reptation for semiflexible actin filaments, Nature (London) 368, 226 (1994).

[2] C. Schuldt, J. Schnauß, T. Händler, M. Glaser, J. Lorenz, T. Golde, J. A. Käs, and D. M. Smith, Tuning Synthetic Semiflexible Networks by Bending Stiffness, Phys. Rev. Lett. 117, 197801 (2016).

[3] M. Keshavarz, H. Engelkamp, J. Xu, O. I. van den Boomen, J. C. Maan, P. C. M. Christianen, and A. E. Rowan, Confining potential as a function of polymer stiffness and concentration in entangled polymer solutions, J. Phys. Chem. B 121, 5613 (2017).

[4] M. Keshavarz, H. Engelkamp, J. Xu, E. Braeken, M. B. J. Otten, H. Uji-i, E. Schwartz, M. Koepf, A. Vananroye, J. Vermant, R. J. M. Nolte, F. De Schryver, J. C. Maan, J. Hofkens, P. C. M. Christianen, and A. E. Rowan, Nanoscale study of polymer dynamics, ACS Nano 10, 1434 (2016).

[5] F. Huber, J. Schnauß, S. Rönicke, P. Rauch, K. Müller, C. Fütterer, and J. A. Käs, Emergent complexity of the cytoskeleton: From single filaments to tissue, Adv. Phys. 62, 1 (2013).

[6] P. Yin, R. F. Hariadi, S. Sahu, H. M. T. Choi, S. H. Park, T. H. LaBean, and J. H. Reif, Programming DNA tube circumferences, Science 321, 824 (2008).

[7] H. Hinsch, J. Wilhelm, and E. Frey, Quantitative tube model for semiflexible polymer solutions, Eur. Phys. J. E 24, 35 (2007).

[8] F. C. MacKintosh, J. Käs, and P. A. Janmey, Elasticity of Semiflexible Biopolymer Networks, Phys. Rev. Lett. 75, 4425 (1995).

[9] H. Isambert and A. C. Maggs, Dynamics and rheology of actin solutions, Macromolecules 29, 1036 (1996).

[10] C. P. Broedersz and F. C. MacKintosh, Modeling semiflexible polymer networks, Rev. Mod. Phys. 86, 995 (2014).

[11] S. F. Edwards, The statistical mechanics of polymerized material, Proc. Phys. Soc. 92, 9 (1967).

[12] P. G. de Gennes, Reptation of a polymer chain in the presence of fixed obstacles, J. Chem. Phys. 55, 572 (1971).

[13] P. G. de Gennes, P. Pincus, R. M. Velasco, and F. Brochard, Remarks on polyelectrolyte conformation, J. Phys. France 37, 1461 (1976).

[14] T. Odijk, The statistics and dynamics of confined or entangled stiff polymers, Macromolecules 16, 1340 (1983).

[15] A. N. Semenov, Dynamics of concentrated solutions of rigid-chain polymers. Part 1 . Brownian motion of persistent macromolecules in isotropic solution, J. Chem. Soc., Faraday Trans. 2 82, 317 (1986).

[16] D. C. Morse, Tube diameter in tightly entangled solutions of semiflexible polymers, Phys. Rev. E 63, 031502 (2001).

[17] M. Tassieri, Dynamics of semiflexible polymer solutions in the tightly entangled concentration regime, Macromolecules 50, 5611 (2017).

[18] T. W. Burkhardt, Free energy of a semiflexible polymer confined along an axis, J. Phys. A: Math. Gen. 28, L629 (1995).

[19] D. Schiffels, T. Liedl, and D. K. Fygenson, Nanoscale structure and microscale stiffness of DNA nanotubes, ACS Nano 7, 6700 (2013).

[20] J. Schnauß, M. Glaser, J. S. Lorenz, C. Schuldt, C. Möser, M. Sajfutdinow, T. Händler, J. A. Käs, and D. M. Smith, DNA nanotubes as a versatile tool to study semiflexible polymers, J. Vis. Exp. 128, 56056 (2017).
[21] M. Glaser, J. Schnauß, T. Tschirner, B. U. S. Schmidt, M. Moebius-Winkler, J. A. Käs, and D. M. Smith, Self-assembly of hierarchically ordered structures in DNA nanotube systems, New J. Phys. 18, 055001 (2016).

[22] H. Isambert, P. Venier, A. C. Maggs, A. Fattoum, R. Kassab, D. Pantaloni, and M. F. Carlier, Flexibility of actin filaments derived from thermal fluctuations. Effect of bound nucleotide, phalloidin, and muscle regulatory proteins, J. Biol. Chem. 270, 11437 (1995).

[23] B. Gentry, D. Smith, and J. Käs, Buckling-induced zebra stripe patterns in nematic F-actin, Phys. Rev. E 79, 031916 (2009).

[24] J. S. Lorenz, J. Schnauß, M. Glaser, M. Sajfutdinow, C. Schuldt, J. A. Käs, and D. M. Smith, Synthetic transient crosslinks program the mechanics of soft, biopolymer-based materials, Adv. Mater. 30, 1706092 (2018).

[25] M. B. Smith, H. Li, T. Shen, X. Huang, E. Yusuf, and D. Vavylonis, Segmentation and tracking of cytoskeletal filaments using open active contours, Cytoskeleton 67, 693 (2010).

[26] G. Marrucci, Relaxation by reptation and tube enlargement: A model for polydisperse polymers, J. Polym. Sci., Polym. Phys. Ed. 23, 159 (1985).

[27] T. Golde, C. Huster, M. Glaser, T. Händler, H. Herrmann, J. A. Käs, and J. Schnauß, Glassy dynamics in composite biopolymer networks, Soft Matter 14, 7970 (2018).

[28] C. F. Schmidt, M. Baermann, G. Isenberg, and E. Sackmann, Chain dynamics, mesh size, and diffusive transport in networks of polymerized actin: A quasielastic light scattering and microfluorescence study, Macromolecules 22, 3638 (1989).

[29] B. Wang, J. Guan, S. M. Anthony, S. C. Bae, Ke. S. Schweizer, and S. Granick, Confining Potential when a Biopolymer Filament Reptates, Phys. Rev. Lett. 104, 118301 (2010).

[30] A. G. Cherstvy, Electrostatic interactions in biological DNA-related systems, Phys. Chem. Chem. Phys. 13, 9942 (2011).

[31] T. Golde, M. Glaser, C. Tutmarc, I. Elbalasy, C. Huster, G. Busteros, D. M. Smith, H. Herrmann, J. A. Käs, and J. Schnauß, The role of stickiness in the rheology of semiflexible polymers, Soft Matter 15, 4865 (2019).

[32] I. Elbalasy, P. Mollenkopf, C. Tutmarc, H. Herrmann, and J. Schnauß, Keratins determine network stress responsiveness in reconstituted actin-keratin filament systems, Soft Matter 17, 3954 (2021).

[33] H. Kubitschke, J. Schnauß, K. D. Nnetu, E. Warmt, R. Stange, and J. Käs, Actin and microtubule networks contribute differently to cell response for small and large strains, New J. Phys. 19, 093003 (2017).

[34] J. Glaser, D. Chakraborty, K. Kroy, I. Lauter, M. Degawa, N. Kirchgeßner, B. Hoffmann, R. Merkel, and M. Giesen, Tube Width Fluctuations in F-Actin Solutions, Phys. Rev. Lett. 105, 037801 (2010).

[35] N. Fakhri, F. C. MacKintosh, B. Lounis, L. Cognet, and M. Pasquali, Brownian motion of stiff filaments in a crowded environment, Science 330, 1804 (2010).

[36] G.J. Papadopoulos and J. Thomchick, On a path integral having application in polymer physics, J. Phys. A: Math. Gen. 10, 1115 (1977).

[37] C. Marques and G. Fredrickson, Rigid Gaussian chains I: The scattering function, J. Phys. II 7, 1805 (1997). 
[38] M. L. Gardel, J. H. Shin, F. C. MacKintosh, L. Mahadevan, P. Matsudaira, and D. A. Weitz, Elastic behavior of cross-linked and bundled actin networks, Science 304, 1301 (2004).

[39] P. Lang and E. Frey, Disentangling entanglements in biopolymer solutions, Nat. Commun. 9, 494 (2018).

[40] T. Golde, C. Schuldt, J. Schnauß, D. Strehle, M. Glaser, and J. Käs, Fluorescent beads disintegrate actin networks, Phys. Rev. E 88, 044601 (2013).
[41] T. Fischer, A. Hayn, and C. T. Mierke, Fast and reliable advanced two-step pore-size analysis of biomimetic 3D extracellular matrix scaffolds, Sci. Rep. 9, 8352 (2019).

[42] M. Čapek, J. Janáček, and L. Kubínová, Methods for compensation of the light attenuation with depth of images captured by a confocal microscope, Microscopy Res. Technique 69, 624 (2006). 